Post-print, please cite as: Heaney, J.L.J., Phillips, A.C., Drayson, M.T. \& Campbell, J. (2016 in press). Serum free lights chains are reduced in endurance trained older adults: evidence that exercise training may reduce basal inflammation in older adults. Experimental Gerontology,

\title{
Serum free lights chains are reduced in endurance trained older adults: evidence that exercise training may reduce basal inflammation in older adults
}

Jennifer L J Heaney ${ }^{\mathrm{a}}$, Anna C Phillips ${ }^{\mathrm{b}}$, Mark T Drayson ${ }^{\mathrm{a}}$, John P Campbell ${ }^{\mathrm{a}}$

${ }^{a}$ Clinical Immunology Service, University of Birmingham, Birmingham, UK

b School of Sport, Exercise and Rehabilitation Sciences, University of Birmingham, Birmingham, UK

Corresponding author:

Jennifer Heaney

Clinical Immunology Service, College of Medical and Dental Sciences, University of Birmingham, Birmingham, West Midlands, UK, B15 2TT

Email: j.l.j.heaney@bham.ac.uk

Accepted refereed manuscript of:

Heaney JLJ, Phillips AC, Drayson MT \& Campbell JP (2016) Serum free light chains are reduced in endurance trained older adults: Evidence that exercise training may reduce basal inflammation in older adults.

Experimental Gerontology, 77, pp. 69-75.

DOI: https://doi.org/10.1016/j.exger.2016.02.011

(C) 2016, Elsevier. Licensed under the Creative Commons Attribution-NonCommercial-NoDerivatives 4.0 International http://creativecommons.org/licenses/by-nc-nd/4.0/ 
Post-print, please cite as: Heaney, J.L.J., Phillips, A.C., Drayson, M.T. \& Campbell, J. (2016 in press). Serum free lights chains are reduced in endurance trained older adults: evidence that exercise training may reduce basal inflammation in older adults. Experimental Gerontology,

http://dx.doi.org/10.1016/i.exger.2016.02.011

\section{Abstract}

Traditionally, free light chains (FLCs) are used as key serum biomarkers in the diagnosis and monitoring of plasma cell malignancies, but polyclonal FLCs can also be used as an accurate real-time indicator of immune-activation and inflammation. The primary aim of the present study was to assess the effects of exercise training status on serum FLCs in older adults, and secondly, to examine if training status moderated serum FLC responses to acute exercise. Kappa and lambda serum FLCs levels were measured in 45 healthy older adults (aged $\geq 60$ years) who were either sedentary, physically active or endurance trained. FLCs were measured at baseline and in response to an acute bout of submaximal exercise. The endurance trained group had significantly lower levels of kappa and lambda serum FLCs compared with physically active or sedentary elderly adults; these effects were independent of age, BMl and renal function. There was no significant difference in whole immunoglobulins between groups. Exercise training status had no effect on serum FLC responses to acute exercise, which were marginal. In conclusion, endurance training was associated with lower FLC levels compared with less physically active individuals. These findings suggest that long-term endurance training may be beneficial in reducing basal inflammation in older adults as well as elevated FLCs present in inflammatory and autoimmune conditions, often associated with ageing. FLCs may serve as a useful biomarker for monitoring the efficacy of exercise intervention studies in healthy and clinical populations.

Keywords: Free light chain, serum, immunoglobulin, exercise, inflammation, older adults 
Post-print, please cite as: Heaney, J.L.J., Phillips, A.C., Drayson, M.T. \& Campbell, J. (2016 in press). Serum free lights chains are reduced in endurance trained older adults: evidence that exercise training may reduce basal inflammation in older adults. Experimental Gerontology,

\section{Introduction}

Light chains make up part of all antibodies (immunoglobulins), however, during plasma cell immunoglobulin synthesis kappa and lambda free light chains (FLCs) are produced in excess of intact immunoglobulin and released into the circulation (Suki and Massry, 1998). Serum FLCs and the ratio of kappa:lambda light chains (K:A ratio) have become key haematological biomarkers in the diagnosis, prognosis and monitoring of plasma cell malignancies (Davids et al., 2010; Dimopoulos et al., 2011; Dispenzieri et al., 2009; Pratt, 2008). Additionally, free light chains have been shown to be intrinsically involved in immune and inflammatory processes (Braber et al., 2012; Brebner and Stockley, 2013; Cohen et al., 2001; Hutchinson et al., 2012 ; Nakano et al., 2011; Redegeld et al., 2012). Indeed, FLCs have been linked to a range of non-malignant inflammatory and autoimmune diseases. Increases in polyclonal FLCs have been published in rheumatoid arthritis, multiple sclerosis, systemic lupus erythematosus, diabetes, and inflammatory bowel disease and may be useful in identifying and monitoring these conditions (Brebner and Stockley, 2013; Nakano et al., 2011; van der Heijden et al., 2006). These studies suggest that polyclonal FLCs may serve as a broad marker of immune activation and inflammation.

Ageing is accompanied by immune and neuroendocrine dysregulation and progression towards low-grade chronic inflammation (inflammaging), which are associated with chronic inflammatory and autoimmune diseases (Baylis et al., 2013; Franceschi and Campisi, 2014; Rehman and Masson, 2001; Weiskopf et al., 2009). Excess polyclonal FLC levels may be another feature of immunosenescence that occurs with age (Dispenzieri et al., 2012) and may serve as a marker of inflammaging. However, excess FLC production may not be a feature of ageing per se; similar ranges of serum FLCs have previously been observed between healthy young and elderly individuals (Heaney et al., 2016). FLCs may be elevated due to immune activation and increased production or through reduced 
Post-print, please cite as: Heaney, J.L.J., Phillips, A.C., Drayson, M.T. \& Campbell, J. (2016 in press). Serum free lights chains are reduced in endurance trained older adults: evidence that exercise training may reduce basal inflammation in older adults. Experimental Gerontology,

http://dx.doi.org/10.1016/i.exger.2016.02.011

metabolism. Due to their clearance through the kidneys, polyclonal FLCs rise with a decline in renal function, which is common in ageing individuals (Hutchinson et al., 2012 ; Weinstein and Anderson, 2010).

Evidence suggests that exercise may be anti-inflammatory in elderly individuals. Higher levels of physical activity have been associated with lower levels of c-reactive protein (CRP) in individuals aged > 65 (Geffken et al., 2001). Further, aerobic exercise training in older adults has been shown to reduced inflammatory markers, CRP, IL-6 and IL-8, compared to flexibility and strength training (Kohut et al., 2006). Studies showing lower pro-inflammatory mediators and improved immune function in trained older adults compared to untrained have been reviewed in detail elsewhere (Simpson et al., 2012). To our knowledge the effect of exercise training on serum FLCs, in either older or young populations, has not yet been explored. The positive benefits of exercise on antiinflammaging may extend to this serological parameter. If so, FLCs could be a useful biomarker for monitoring the efficacy of exercise intervention studies in healthy and clinical populations.

Recent studies have investigated the effect of acute exercise on serum FLCs. Kappa FLCs have been shown to be marginally elevated in runners following a marathon compared with pre-exercise levels, thought to be due to an acute and transient decline in renal function proportional to exercise intensity (Campbell et al., 2016). Although no changes in serum FLCs were seen in elderly individuals who undertook a 4-day walking exercise (Jacobs et al., 2014) the effect of training status - i.e., sedentary versus physical active - was not investigated. Training load has previously been shown to influence baseline renal function (Touchberry et al., 2004) and thus may impact upon FLC clearance during acute exercise; to date, this has not been investigated in younger or older adults. It is possible that individuals unaccustomed to exercise may be more vulnerable to transient 
Post-print, please cite as: Heaney, J.L.J., Phillips, A.C., Drayson, M.T. \& Campbell, J. (2016 in press). Serum free lights chains are reduced in endurance trained older adults: evidence that exercise training may reduce basal inflammation in older adults. Experimental Gerontology,

http://dx.doi.org/10.1016/i.exger.2016.02.011

perturbations in renal function, and consequently FLCs, with an acute bout of exercise.

The primary aim of the present study was to assess the effects of exercise training status

on serum FLCs in older adults, and secondly, to examine if training status moderates serum FLC responses to acute exercise.

\section{Methods}

\subsection{Participants}

Participants were community dwelling older adults ( $n=45,27$ males) aged $67.25 \pm 5.06$ years old from the UK. Twenty percent of participants reported suffering from a chronic illness, which were hypertension and asthma, and $38 \%$ reported taking medication, such as anti-hypertensives, non-corticosteroid inhalers, statins and gastrointestinal medications. Participants were excluded if suffering from any immune or endocrine disorder or any condition that precluded them from exercise, or if they had suffered from any acute illness in the two weeks prior to, or during the study.

\subsection{Exercise groups}

Participants were either sedentary $(n=13)$, physically active $(n=16)$, or endurance trained $(n=16)$. The sedentary participants were not currently involved in any regular exercise nor had they been for 5 years prior to the study; physically active participants regularly engaged in activities that were of moderate intensity: rambling, keep fit classes, swimming or badminton; the endurance trained older athletes were competitive runners, some of whom also cycled or took part in circuit training. Participants were given a 14 day exercise diary to assess how long each exercise group spent doing moderate and vigorous activities. They were asked to record the type, duration and intensity of the activity. The intensity was determined using a $0-10$ version of Rating of Perceived Exertion (RPE) scale (Borg, 1998), where 0 is rest and 10 was maximal effort. 
Post-print, please cite as: Heaney, J.L.J., Phillips, A.C., Drayson, M.T. \& Campbell, J. (2016 in press). Serum free lights chains are reduced in endurance trained older adults: evidence that exercise training may reduce basal inflammation in older adults. Experimental Gerontology,

http://dx.doi.org/10.1016/i.exger.2016.02.011

Participants were briefed on the use of the RPE Scale and instructions and examples were also provided in the diary. Time spent in moderate and vigorous activity was calculated based upon metabolic equivalents for specific activities (Ainsworth et al., 2000) and RPE (Nelson et al., 2007). Minutes at each intensity level over the 14 day period were averaged per week.

\subsection{Exercise protocol}

Participants arrived to the laboratory between 08:00 and 09:00 where they were fitted with a HR monitor (Polar, Electro Kempele, Finland). They were then familiarised with the procedure for collection of expired gas using Douglas bags. They then rested for $15 \mathrm{~min}$ before a blood sample was taken. Participants then completed an acute bout of exercise in the form of an incremental submaximal treadmill test. Participants began walking on the treadmill and speed was gradually increased until the participant reached a pace they considered 'brisk walking'. The gradient was increased every 4 min and during the final min of each stage expired gas samples were collected into Douglas bags (Cranlea, Birmingham, UK). HR was monitored continuously throughout the exercise and the test was terminated once the participant had reached $75 \%$ of their predicted maximum $\mathrm{HR}$, as determined by the formula: 208-(age×0.7) (Tanaka et al., 2001). A blood sample was taken immediately after exercise and $1 \mathrm{~h}$ post-exercise. Between post- and $1 \mathrm{~h}$ postexercise participants were allowed to drink water ad libitum. $\mathrm{VCO}_{2}$ and $\mathrm{VO}_{2}$ were determined using $\mathrm{O}_{2} / \mathrm{CO}_{2}$ analyser (Servomex 1440, Crowborough, UK) and expired gas volumes were measured using a dry gas meter (Harvard Apparatus, Edenbridge, UK). Using a regression equation created from plotting the relationship between $\mathrm{HR}$ and $\mathrm{VO}_{2}$ during the final three stages of exercise, $\mathrm{VO}_{2 \max }$ was predicted. 
Post-print, please cite as: Heaney, J.L.J., Phillips, A.C., Drayson, M.T. \& Campbell, J. (2016 in press). Serum free lights chains are reduced in endurance trained older adults: evidence that exercise training may reduce basal inflammation in older adults. Experimental Gerontology,

\subsection{Blood samples and analyses}

Venous blood was collected from an antecubital vein into plain tubes (BD Vacutainer, Plymouth, UK) pre-, post- and $1 \mathrm{~h}$ post-exercise. Blood was allowed to clot at room temperature before being centrifuged and the separated serum was stored at $-20^{\circ} \mathrm{C}$ until analysis. Serum kappa and lambda FLCs were quantified using a multi-plex bead-based assay using a Luminex platform (Bio-plex systems, BioRad Laboratories, California, USA). This assay was developed by the Clinical Immunology Service at the University of Birmingham and uses mAbs specific for either kappa or lambda FLC (Abingdon Health Ltd, Oxford, UK) in a competitive inhibition format. Full details for this assay have been described previously (Campbell et al., 2013). Serum creatinine, CRP, IgG, IgA and IgM were measured on a Roche Hitachi Modular. To minimize variation, all Roche Hitachi Modular analyses were performed on a single day using the same calibration set-up, batches, and user. Plasma volume changes as a result of exercise were estimated between sample collection time points (Dill and Costill, 1974).

\subsection{Free light chain parameters}

A range of FLC parameter outputs were analysed as part of this study. In addition to concentrations of kappa and lambda FLC, the K: $\lambda$ ratio and the difference between kappa and lambda FLC (FLC difference) were examined. The $\mathrm{K}: \lambda$ ratio and FLC difference are classically used in conditions involving perturbed levels of FLC, such as plasma cell disorders (Dispenzieri et al., 2008; Siegel et al., 2009). The sum of kappa and lambda FLCs (FLC sum) was also investigated as this measure has been employed in the general population who do not have clonal plasma cell disease (Dispenzieri et al., 2012). All the above parameters were included to enable any future comparisons between healthy and clinical populations. Participant's serum FLC results were screened at baseline to exclude any patients with abnormal serum samples suggestive of undiagnosed monoclonal 
Post-print, please cite as: Heaney, J.L.J., Phillips, A.C., Drayson, M.T. \& Campbell, J. (2016 in press). Serum free lights chains are reduced in endurance trained older adults: evidence that exercise training may reduce basal inflammation in older adults. Experimental Gerontology,

http://dx.doi.org/10.1016/i.exger.2016.02.011

gammopathy of unknown significance (MGUS), immune stimulation or suppression or renal impairment. The most widely cited reference ranges for FLCs are $3.30-19.40 \mathrm{mg} / \mathrm{L}$ for kappa, $5.7-26.30 \mathrm{mg} / \mathrm{L}$ for lambda and $0.26-1.65$ for the $\mathrm{K}: \lambda$ ratio (Katzmann et al., 2002). The references ranges for the Luminex serum FLC used in this study were very similar: $4.35-19.44 \mathrm{mg} / \mathrm{L}$ for kappa, $5.19-18.87 \mathrm{mg} / \mathrm{L}$ for lambda and $0.40-1.59$ for the $\mathrm{K}: \lambda$ ratio (Campbell et al., 2013). Serum FLC results at baseline were compared to both sets of reference ranges and anyone outside either of these ranges were excluded from the final analyses.

\subsection{Statistical analyses}

Analyses were undertaken using IBM SPSS version 21. Renal function was assessed by calculating estimated creatinine clearance rates $(\mathrm{eCrCl})$ and estimated glomerular filtration rate (eGFR) using the Cockcroft-Gault ([140-age] x mass $x$ constant [1.23 for men or 1.04 for women]/serum creatinine) and Modification of Diet in Renal Disease (186 x serum creatinine $e^{-1.154} \times$ age ${ }^{-0.203} \times 0.742$ if female) formulas, respectively. Univariate ANOVA was used to assess differences between exercise groups in: age, BMI, time spent in moderate/vigorous activity, $\mathrm{VO}_{2 \max }$ and baseline renal function. Chi-square was used to assess group differences in categorical variables: sex and chronic medication usage. Repeated measures ANOVA was used to analyse responses to exercise, to test any main effects of exercise group and assess any group $\mathrm{x}$ time interactions for all serological variables: FLC parameters (kappa and lambda concentrations, K: $\lambda$ ratio, FLC difference, FLC sum), IgG, IgA, IgM, creatinine and CRP. Percentage change in serological parameters were calculated between pre- and post-exercise time points and compared between exercise groups using univariate ANOVA. When significant main effects of time or group were observed, Bonferroni post-hoc tests were applied. Subsequent repeated measures/univariate ANCOVAs were performed for significant findings to control for any 
Post-print, please cite as: Heaney, J.L.J., Phillips, A.C., Drayson, M.T. \& Campbell, J. (2016 in press). Serum free lights chains are reduced in endurance trained older adults: evidence that exercise training may reduce basal inflammation in older adults. Experimental Gerontology,

http://dx.doi.org/10.1016/i.exger.2016.02.011

confounding effects of age and BMI in relation to effects of group, or plasma volume change in relation to effects of exercise. Slight variations in degrees of freedom reflect occasional missing data or insufficient serum for analysis of all parameters. Pearson's correlation was used to examine the relationship between FLC parameters and immunoglobulins, CRP and creative at baseline and percentage change in response to exercise between FLCs and other serum parameters. Greenhouse-Geisser corrected $F$ values are reported for repeated measures analyses and partial $\eta^{2}$, a measure of effect size, is reported throughout.

\section{Results}

\subsection{Baseline serology screening}

In order to exclude any individuals with abnormal serum samples, participant's serum FLC results were screened at baseline and compared to the normal reference ranges outlined in the Methods. Four individuals exhibited abnormal $\mathrm{K}: \lambda$ ratios at baseline, and were consequently excluded from the study analyses. Three of these individuals had elevated kappa or lambda FLCs, indicative of possible MGUS. The other individual had very low levels of FLCs, suggestive of potential immunosuppression. All other participants had kappa and lambda free light chain concentrations and $\mathrm{K}: \lambda$ ratios within the normal range. Serology results at baseline for all final participants included in the study and their respective exercise group are shown in Table 1. A total of 41 participants were included for all subsequent analyses: 11 sedentary, 16 physically active and 14 endurance trained individuals. Creatinine levels, eGrCl and eGFR (Table 1), indicators of renal function, did not significantly differ between exercise groups and therefore were not used as covariates for subsequent FLC analyses. 
Post-print, please cite as: Heaney, J.L.J., Phillips, A.C., Drayson, M.T. \& Campbell, J. (2016 in press). Serum free lights chains are reduced in endurance trained older adults: evidence that exercise training may reduce basal inflammation in older adults. Experimental Gerontology,

\subsection{Participants characteristics}

Participant characteristics are displayed in Table 2. Age $\left(F(2,38)=3.50, p=.040, \eta^{2}=\right.$ $.156)$ and $\mathrm{BMI}\left(F(2,38)=5.67, p=.007, \eta^{2}=.230\right)$ significantly differed between exercise groups; the endurance group was significantly younger compared with the physically active group, but not sedentary adults. As anticipated, BMI was lower in endurance trained athletes compared with sedentary participants, although the endurance trained and physically active did not significantly differ in BMI. The distribution of males and females between exercise groups did not significantly differ. As expected, exercise participation (exercise diary results) varied between groups. The physically active group engaged in a significantly higher amount of moderate activity per week compared with the other groups, $F(2,34)=22.14, p<.001, \eta^{2}=.566$. The endurance trained group spent significantly more time participating in vigorous activity compared with the sedentary and physically active groups, $F(2,34)=30.37, p<.001, \eta^{2}=.641$, and had a significantly higher $\mathrm{VO}_{2 \max }$ compared with the other groups, $F(2,32)=24.26, p<.001, \eta^{2}=.603$. The distribution of individuals with chronic illness or taking chronic medication did not differ between exercise groups, nor was there any difference between FLC parameters based upon chronic illness or medication status; therefore these parameters were not included as covariates in subsequent analysis.

\subsection{Acute exercise parameters}

The mean exercise duration of the submaximal exercise bout was $23 \mathrm{~min} 24 \mathrm{sec} \pm 3 \mathrm{~min}$ $18 \mathrm{sec}$ and the final RPE obtained at the end of the exercise was $5.23 \pm 1.05$ ("hard"). The mean maximum heart rate achieved was $119.64 \pm 40.80 \mathrm{bpm}$, equivalent to $76.50 \pm 4.86$ $\%$ predicted maximum HR. There were no significant differences for any of these exercise parameters between the exercise groups. 
Post-print, please cite as: Heaney, J.L.J., Phillips, A.C., Drayson, M.T. \& Campbell, J. (2016 in press). Serum free lights chains are reduced in endurance trained older adults: evidence that exercise training may reduce basal inflammation in older adults. Experimental Gerontology,

http://dx.doi.org/10.1016/i.exger.2016.02.011

Overall, the mean \pm SD plasma volume reduction post-exercise was $-4.84 \pm 5.12 \%$ and by

$1 \mathrm{~h}$ post-exercise had increased above pre-exercise levels by $3.45 \pm 4.55 \%$. Plasma

volume increased by a mean of $8.80 \pm 5.26 \%$ between immediately post- and $1 \mathrm{~h}$ post-

exercise, reflecting ad libitum drinking after the exercise. There was no significant difference between training status groups for plasma volume changes.

\subsection{The effect of training status and acute exercise on free light chains}

Among the older adults, those who were endurance trained demonstrated significantly lower kappa (main effect of group: $F(2,38)=5.57, p=.008, \eta^{2}=.227$ ) and lambda FLCs (main effect of group: $F(2,38)=8.63, p=.001, \eta^{2}=.312$ ) in serum compared with moderately trained and sedentary groups (Figure 1). As kappa and lambda light chains were significantly lower in the endurance trained group, the FLC sum was also significantly lower; main effect of group, $F(2,38)=8.16, p=.001, \eta^{2}=.300$. Values for these parameters were lower at baseline and remained consistently lower across time points, with no group $\mathrm{x}$ time interactions in response to acute exercise. There was a significant group $\mathrm{x}$ time interaction for the $\mathrm{K}: \lambda$ ratio, whereby endurance trained individuals had a significantly higher ratio pre-exercise compared to the other groups $(F(4,76)=8.09, p<$ $.001, \eta^{2}=.299$ ). This was driven by higher kappa levels relative to lambda levels in the endurance trained group at this time point, illustrated by a significantly higher FLC difference pre-exercise, $F(4,76)=4.47, p=.003, \eta^{2}=.190$. These findings withstood adjustment for age and BMI. Descriptive statistics for FLC parameters between exercise groups at baseline are displayed in Table 1. No sex differences were observed for any FLC parameters.

Mean percentage changes for FLCs in response to exercise for the sample as a whole were small: mean \pm SD kappa \% change was $0.22 \pm 7.75 \%$ and $-0.36 \pm 7.29 \%$; mean 
Post-print, please cite as: Heaney, J.L.J., Phillips, A.C., Drayson, M.T. \& Campbell, J. (2016 in press). Serum free lights chains are reduced in endurance trained older adults: evidence that exercise training may reduce basal inflammation in older adults. Experimental Gerontology,

http://dx.doi.org/10.1016/i.exger.2016.02.011

lambda \% change was $1.18 \pm 8.51 \%$ and $-0.87 \pm 9.26 \%$, for pre- to post-exercise and preto $1 \mathrm{~h}$ post-exercise, respectively. Although no significant group $\mathrm{x}$ time interactions were observed for mean FLC parameters in response to exercise, percentage changes did produce significant findings between groups. As shown in Table 3, kappa FLCs significantly reduced between pre- to post-exercise in endurance trained athletes compared with the physically active group, $F\left(2,38=3.80, p=.031, \eta^{2}=.167\right)$. Alternatively, for lambda FLCs, endurance athletes demonstrated a significant percentage increase post-exercise compared to the sedentary group, $F(2,38)=4.25, p=.022, \eta^{2}=$ .183. These group differences in percentage change were reduced to trends when controlling for either BMI (lambda, $\mathrm{p}=.065$ ) or age (kappa, $p=.080$ ). As these findings were in response to exercise, plasma volume changes were also included as a potential confounding variable; the group difference in lambda remained significant $(p=.040)$ although the kappa finding was reduced to a trend $(p=.056)$. No significant differences were observed between training groups for percentage change between pre- and $1 \mathrm{~h}$ postexercise.

\subsection{The effect of exercise training status and acute exercise on immunoglobulin levels} A significant main effect of time was observed for IgG, where values significantly increased post-exercise, followed by a significant decrease between post- and $1 \mathrm{~h}$ post-exercise, $F(2,74)=26.79, p<.001, \eta^{2}=.420$. The same response pattern was observed for $\lg A$ and $\operatorname{lgM}, F(2,74)=41.11, p<.001, \eta^{2}=.526$ and $F(2,74)=23.21, p<.001, \eta^{2}=.386$, respectively. These significant changes in response to exercise are shown in Figure 2. For IgG and IgA, these effects of time withstood adjustment for plasma volume change for pre- to post-exercise increases, but reductions between post- and $1 \mathrm{~h}$-post exercise were no longer significant; IgM findings were no longer significant when adding plasma volume 
Post-print, please cite as: Heaney, J.L.J., Phillips, A.C., Drayson, M.T. \& Campbell, J. (2016 in press). Serum free lights chains are reduced in endurance trained older adults: evidence that exercise training may reduce basal inflammation in older adults. Experimental Gerontology,

http://dx.doi.org/10.1016/i.exger.2016.02.011

change as a covariate. No significant main effect of group or group $\mathrm{x}$ time interactions occurred for immunoglobulins.

Immunoglobulins increased by similar proportion between pre- and post-exercise: $4.85 \%$, $5.42 \%$ and $4.79 \%$ for $\lg G$, IgA and $\lg M$, respectively. The same consistency was observed across heavy chain subtypes for decreases between post- and $1 \mathrm{~h}$ postexercise: $-4.71 \%$ for $\lg G,-5.11 \%$ for $\lg A$ and $-5.20 \%$ for $\lg M$. Percentage changes in immunoglobulins in response to exercise for the different exercise groups are shown in Table 3. No significant differences occurred in immunoglobulin percentage changes between exercise groups.

3.5. The effect of exercise training status and acute exercise on creatinine levels and renal function

As shown in Figure 3, creatinine decreased from post- to 1 h post-exercise, $F(2,74)=4.66$, $p=.012, \eta^{2}=.112$. This decrease was equal to $-3.67 \pm 7.22 \%$ and levels at $1 \mathrm{~h}$ were below pre-exercise values. As a result of this reduction in creatinine, $\mathrm{e} \operatorname{GrCl}(F(2,74)=$ 4.17, $\left.p=.019, \eta^{2}=.112\right)$ and eGFR $\left(F(2,74)=3.91, p=.024, \eta^{2}=.096\right)$ increased between these timepoints. Upon controlling for plasma volume change between these time points, these main effects of time were no longer significant. No effects of group or group $\mathrm{x}$ time interactions were observed. Percentage changes in creatinine and renal function responses to exercise for the different exercise groups are shown in Table 3.

\subsection{The effect of exercise training status and acute exercise on CRP levels}

The sedentary group had higher CRP at baseline (Table 1), although this was not statistically significant. A significant main effect of time occurred for CRP, $F(2,74)=10.05$, $p<.001, \eta^{2}=.214$ (Figure 3). A significant increase in CRP was observed post-exercise by $6.11 \pm 10.43 \%$, followed by a decrease between post- and $1 \mathrm{~h}$ post-exercise by $-7.81 \pm$ 
Post-print, please cite as: Heaney, J.L.J., Phillips, A.C., Drayson, M.T. \& Campbell, J. (2016 in press). Serum free lights chains are reduced in endurance trained older adults: evidence that exercise training may reduce basal inflammation in older adults. Experimental Gerontology, http://dx.doi.org/10.1016/i.exger.2016.02.011

$7.21 \%$, returning close to pre-exercise values. The pre- to post-exercise increase remained significant when controlling for plasma volume change, but the decrease post- to $1 \mathrm{~h}$ post-exercise did not withstand adjustment. There were no group or group $\mathrm{x}$ time interactions for CRP, nor were there any difference in percentage change in CRP in response to exercise between groups (Table 3).

\subsection{The relationship between free light chains and other serological parameters}

As expected, at baseline, kappa FLCs correlated significantly with $\lg G, r(38)=.480, p=$ $0.002, \lg \mathrm{A}, r(38)=.475, p=.002$, and $\lg \mathrm{M}, r(38)=.431, p=.005$. Similarly, lambda FLCs at baseline correlated with $\lg G, r(38)=.409, p=.002$, $\lg \mathrm{A}, r(38)=.467, p=.009$, and $\lg \mathrm{M}$ $, r(38)=.359, p=.023$. No significant relationships existed between kappa or lambda FLC parameters with creatinine or CRP. Percentage changes in response to exercise in kappa or lambda FLCs did not correlate with percentage changes in immunoglobulins, creatinine or CRP.

\section{Discussion}

The present study demonstrates that endurance trained older adults have significantly lower levels of kappa and lambda serum FLCs compared with elderly adults who are physically active or sedentary. These group differences in FLCs levels and consequently FLC sum were observed at baseline and throughout acute exercise, and were independent of age, BMI and renal function. Further, these results are unlikely to be due to reverse causation, i.e. individuals with illness show elevated FLCs and are less able to exercise, given that participants were excluded if suffering from any immune or endocrine disorder or any condition that precluded them from exercise. Due to the role of FLCs in 
Post-print, please cite as: Heaney, J.L.J., Phillips, A.C., Drayson, M.T. \& Campbell, J. (2016 in press). Serum free lights chains are reduced in endurance trained older adults: evidence that exercise training may reduce basal inflammation in older adults. Experimental Gerontology,

http://dx.doi.org/10.1016/i.exger.2016.02.011

immune and inflammatory processes, these findings suggest that long-term endurance training may reduce basal inflammation in older adults.

Previous cross-sectional studies have shown that exercise trained older adults have lower levels of inflammatory markers and better immune function compared with less active/sedentary individuals (Geffken et al., 2001; Kohut and Senchina, 2004; Simpson et al., 2012; Woods et al., 2012). Evidence from intervention studies have also been reviewed, and, in general, aerobic exercise programmes of $\geq 6$ months are able to reduce inflammatory markers and improve immune function in the elderly (Kohut and Senchina, 2004; Woods et al., 2012). The present study extends the potential anti-inflammatory effects of exercise in older adults to FLCs. It is also worth noting that CRP was lower in the physically active and endurance trained groups compared to those who were sedentary, although this difference was not significant. This could be a result of the modest sample size of this study or potentially FLCs could be a more sensitive marker than CRP in relation to exercise training. As elevated polyclonal FLCs may be a characteristic of immunosenescence (Dispenzieri et al., 2012), the lower FLCs in the endurance trained individuals may be indicative of a slower rate of immune dysregulation and lower inflammatory profile with long-term regular exercise. It should be remembered that this is a relatively small initial study in this area of research and larger investigations should consider FLCs in conjunction with a range of immune parameters and other markers of inflammation. Additionally, intervention studies are required to establish if exercise (and what amount of exercise) can reduce FLCs in older adults presenting with elevated FLCs, in individuals suffering from inflammatory or autoimmune disease or haematological malignancies.

The mechanism linking exercise and serum FLC levels is unknown. By nature, the endurance trained participants in the present study had a lower BMI compared to the 
Post-print, please cite as: Heaney, J.L.J., Phillips, A.C., Drayson, M.T. \& Campbell, J. (2016 in press). Serum free lights chains are reduced in endurance trained older adults: evidence that exercise training may reduce basal inflammation in older adults. Experimental Gerontology,

http://dx.doi.org/10.1016/i.exger.2016.02.011

sedentary group, however, FLC differences withstood adjustment for BMI suggesting group diffrences were independent of potential inflammatory effects of adipose tissue in those less active. There may be a direct effect of exercise on FLC levels through secretion rates and/or renal clearance, alternatively the relationship may best viewed in context of other inflammatory and immune changes that take place with exercise. Importantly, there was no difference in whole immunoglobulin levels between exercise groups. However, the higher levels of FLCs observed in sedentary and physically active groups do not appear to be due to impaired renal clearance, given that indicators of renal function were comparable across groups. It is possible that plasma cells are secreting the same amount of whole immunoglobulin, but lower amounts of FLC in the endurancetrained group. Taken together, these suppositions need to be addressed in future studies to understand the mechanism of association between exercise and FLCs.

Higher levels of polyclonal FLCs have previously been associated with survival. Dispenzeri et al (2012) demonstrated that individuals from the general population with the highest levels of FLCs were at greater risk of death. The potential of serum FLCs as a predictive biomarker has also been shown in a hospital referral population; elevated FLCs were associated with an increased risk of mortality over 4.5 years (Anandram et al., 2012). However, it has yet to be established if the relationship between FLCs and disease and mortality is causative or a proxy marker for underlying diseases processes (Dispenzieri et al., 2012). From this cross-sectional study it cannot be determined if the lower levels of FLCs in endurance trained older adults may be protective against risk of adverse events. Further, it should be noted that all participants in this study had FLC levels within the normal range and those who were endurance trained were engaging in what could be considered to be a high levels of vigorous exercise for this age group (on average nearly 4 hours per week). Longitudinal studies with a larger number of participants will enable broader ranges of polyclonal FLC levels and training volumes to be captured and are 
Post-print, please cite as: Heaney, J.L.J., Phillips, A.C., Drayson, M.T. \& Campbell, J. (2016 in press). Serum free lights chains are reduced in endurance trained older adults: evidence that exercise training may reduce basal inflammation in older adults. Experimental Gerontology,

http://dx.doi.org/10.1016/i.exger.2016.02.011

required to establish if exercise behaviour and FLCs interact to predict the risk of inflammatory disease or mortality.

There were no significant overall effects of acute exercise on FLCs, with mean changes < $\pm 1.5 \%$ between time points. This is consistent with prior investigations into acute exercise in both older adults (Jacobs et al., 2014) and young adults (Heaney et al., 2016) suggesting FLC production is generally not affected by exercise. Conversely, marathon running has been shown to elicit modest elevations in Kappa FLCs proportional to exercise intensity (Campbell et al., 2016). Therefore exercise may have to be very intense and prolonged to induce changes in FLC levels. Training status had marginal effects on percentage changes in FLCs in response to exercise, which emerged only as trends when controlling for confounding variables. Consequently, the hypothesis that training status moderates FLC responses to acute exercise should be rejected.

Serum immunoglobulins showed moderate correlations with FLC levels at baseline, although the training effect observed with FLCs was not replicated for $\lg G$, IgA or IgM. In addition, there were no differences in immunoglobulin responses to exercise between groups; these findings are consistent with previous reports (Nieman and NehlsenCannarella, 1991). Immunoglobulins significantly increased immediately post-exercise, in agreement with recent findings (Campbell et al., 2016). Levels then decreased between post- and $1 \mathrm{~h}$ post-exercise, reflecting increases in plasma volume due to rehydration through ad libitum water consumption during this time frame. Interestingly, despite most likely having poorer renal function relative to younger adults, this type of graded submaximal exercise did not cause a spike in serum creatinine in this elderly cohort; an effect seen previously with more intense/prolonged exercise (McCullough et al., 2011).

\subsection{Conclusions}


Post-print, please cite as: Heaney, J.L.J., Phillips, A.C., Drayson, M.T. \& Campbell, J. (2016 in press). Serum free lights chains are reduced in endurance trained older adults: evidence that exercise training may reduce basal inflammation in older adults. Experimental Gerontology,

http://dx.doi.org/10.1016/i.exger.2016.02.011

In this cross-sectional study of sedentary, physically active and endurance trained older adults aged $\geq 60$ years, kappa and lambda serum FLC levels, and consequently the FLC sum, were found to be significantly lower in endurance trained individuals. Acute exercise had negligible effects on FLCs and responses to exercise were not affected by training status. This is the first time that FLCs have been examined in response to exercise habitual behaviour; these preliminary findings build upon previous evidence that exercise training may have an anti-inflammatory effect in older adults. Exercise may be able to attenuate not only inflammaging but may be beneficial in reducing FLC levels in inflammatory and autoimmune conditions associated with elevated polyclonal FLCs, which are prevalent in older populations. This study highlights the potential utility of FLCs as a biomarker in ageing and health research and suggests that FLCs may be useful in monitoring the efficacy of interventions aimed at reducing chronic inflammation, such as exercise. 
Post-print, please cite as: Heaney, J.L.J., Phillips, A.C., Drayson, M.T. \& Campbell, J. (2016 in press). Serum free lights chains are reduced in endurance trained older adults: evidence that exercise training may reduce basal inflammation in older adults. Experimental Gerontology,

\section{References}

Ainsworth, B.E., Haskell, W.L., Whitt, M.C., Irwin, M.L., Swartz, A.M., Strath, S.J., O'Brien, W.L., Bassett, D.R., Jr., Schmitz, K.H., Emplaincourt, P.O., Jacobs, D.R., Jr., Leon, A.S. 2000. Compendium of physical activities: an update of activity codes and MET intensities. Med Sci Sports Exerc 32, S498-504.

Anandram, S., Assi, L.K., Lovatt, T., Parkes, J., Taylor, J., Macwhannell, A., Jacob, A., Handa, S., Harding, S., Basu, S. 2012. Elevated, combined serum free light chain levels and increased mortality: a 5-year follow-up, UK study. J Clin Pathol 65, 1036-1042.

Baylis, D., Bartlett, D.B., Patel, H.P., Roberts, H.C. 2013. Understanding how we age: insights into inflammaging. Longev Healthspan 2, 2046-2395.

Borg, G.A., 1998. Borg's ratings of percieved exhertion and pain scales. Human Kinetics, Champaign.

Braber, S., Thio, M., Blokhuis, B.R., Henricks, P.A., Koelink, P.J., Groot Kormelink, T., Bezemer, G.F., Kerstjens, H.A., Postma, D.S., Garssen, J., Kraneveld, A.D., Redegeld, F.A., Folkerts, G. 2012. An association between neutrophils and immunoglobulin free light chains in the pathogenesis of chronic obstructive pulmonary disease. Am J Respir Crit Care Med 185, 817-824.

Brebner, J.A., Stockley, R.A. 2013. Polyclonal free light chains: a biomarker of inflammatory disease or treatment target? F1000 Med Rep 5, 1.

Campbell, J.P., Cobbold, M., Wang, Y., Goodall, M., Bonney, S.L., Chamba, A., Birtwistle, J., Plant, T., Afzal, Z., Jefferis, R., Drayson, M.T. 2013. Development of a highly-sensitive multiplex assay using monoclonal antibodies for the simultaneous measurement of kappa and lambda immunoglobulin free light chains in serum and urine. J Immunol Methods 391, 1-13.

Campbell, J.P., Eijsvogels, T.M.H., Wang, Y., Hopman, M.T.E., Drayson, M.T., Jacobs, J.F. 2016. Changes to serum free light chain levels in healthy adults immediately after marathon running. Clin Chem Lab Med 54, 459-465. 
Post-print, please cite as: Heaney, J.L.J., Phillips, A.C., Drayson, M.T. \& Campbell, J. (2016 in press). Serum free lights chains are reduced in endurance trained older adults: evidence that exercise training may reduce basal inflammation in older adults. Experimental Gerontology,

http://dx.doi.org/10.1016/i.exger.2016.02.011

Cohen, G., Rudnicki, M., Horl, W.H. 2001. Uremic toxins modulate the spontaneous apoptotic cell death and essential functions of neutrophils. Kidney Int Suppl 78, S48-52.

Davids, M.S., Murali, M.R., Kuter, D.J. 2010. Serum free light chain analysis. Am J Hematol 85, 787-790.

Dill, D.B., Costill, D.L. 1974. Calculation of percentage changes in volumes of blood, plasma, and red cells in dehydration. J Appl Physiol 37, 247-248.

Dimopoulos, M., Kyle, R., Fermand, J.P., Rajkumar, S.V., San Miguel, J., Chanan-Khan, A., Ludwig, H., Joshua, D., Mehta, J., Gertz, M., Avet-Loiseau, H., Beksac, M., Anderson, K.C., Moreau, P., Singhal, S., Goldschmidt, H., Boccadoro, M., Kumar, S., Giralt, S., Munshi, N.C., Jagannath, S. 2011. Consensus recommendations for standard investigative workup: report of the International Myeloma Workshop Consensus Panel 3. Blood 117, 4701-4705.

Dispenzieri, A., Katzmann, J.A., Kyle, R.A., Larson, D.R., Therneau, T.M., Colby, C.L., Clark, R.J., Mead, G.P., Kumar, S., Melton, L.J., 3rd, Rajkumar, S.V. 2012. Use of nonclonal serum immunoglobulin free light chains to predict overall survival in the general population. Mayo Clin Proc 87, 517-523.

Dispenzieri, A., Kyle, R., Merlini, G., Miguel, J.S., Ludwig, H., Hajek, R., Palumbo, A., Jagannath, S., Blade, J., Lonial, S., Dimopoulos, M., Comenzo, R., Einsele, H., Barlogie, B., Anderson, K., Gertz, M., Harousseau, J.L., Attal, M., Tosi, P., Sonneveld, P., Boccadoro, M., Morgan, G., Richardson, P., Sezer, O., Mateos, M.V., Cavo, M., Joshua, D., Turesson, I., Chen, W., Shimizu, K., Powles, R., Rajkumar, S.V., Durie, B.G. 2009. International Myeloma Working Group guidelines for serum-free light chain analysis in multiple myeloma and related disorders. Leukemia 23, 215-224.

Dispenzieri, A., Zhang, L., Katzmann, J.A., Snyder, M., Blood, E., Degoey, R., Henderson, K., Kyle, R.A., Oken, M.M., Bradwell, A.R., Greipp, P.R. 2008. Appraisal of immunoglobulin free light chain as a marker of response. Blood 111, 4908-4915.

Franceschi, C., Campisi, J. 2014. Chronic inflammation (inflammaging) and its potential contribution to age-associated diseases. J Gerontol A Biol Sci Med Sci 69, S4-9. 
Post-print, please cite as: Heaney, J.L.J., Phillips, A.C., Drayson, M.T. \& Campbell, J. (2016 in press). Serum free lights chains are reduced in endurance trained older adults: evidence that exercise training may reduce basal inflammation in older adults. Experimental Gerontology,

Geffken, D.F., Cushman, M., Burke, G.L., Polak, J.F., Sakkinen, P.A., Tracy, R.P. 2001.

Association between physical activity and markers of inflammation in a healthy elderly population. Am J Epidemiol 153, 242-250.

Heaney, J.L.J., Gleeson, M., Phillips, A.C., Taylor, I.M., Drayson, M.T., Goodall, M., He, C.-S., Svendsen, I.S., Killer, S.C., Campbell, J.P. 2016. Salivary immunoglobulin free light chains: reference ranges and responses to exercise in young and older adults. In submission Exerc Immunol Rev, 28-41.

Hutchinson, A.T., Jones, D.R., Raison, R.L. 2012 The ability to interact with cell membranes suggests possible biological roles for free light chain. Immunol Lett 142, 75-77.

Jacobs, J.F., Eijsvogels, T.M., van der Geest, K.S., Koenen, H.J., Hutchison, C.A., Boots, A.M., Hopman, M.T., Joosten, I. 2014. The impact of exercise on the variation of serum free light chains. Clin Chem Lab Med 52, e239-242.

Katzmann, J.A., Clark, R.J., Abraham, R.S., Bryant, S., Lymp, J.F., Bradwell, A.R., Kyle, R.A. 2002. Serum reference intervals and diagnostic ranges for free kappa and free lambda immunoglobulin light chains: relative sensitivity for detection of monoclonal light chains. Clin Chem 48, 1437-1444.

Kohut, M.L., McCann, D.A., Russell, D.W., Konopka, D.N., Cunnick, J.E., Franke, W.D., Castillo, M.C., Reighard, A.E., Vanderah, E. 2006. Aerobic exercise, but not flexibility/resistance exercise, reduces serum IL-18, CRP, and IL-6 independent of beta-blockers, BMI, and psychosocial factors in older adults. Brain Behav Immun 20, 201-209.

Kohut, M.L., Senchina, D.S. 2004. Reversing age-associated immunosenescence via exercise. Exerc Immunol Rev 10, 6-41.

McCullough, P.A., Chinnaiyan, K.M., Gallagher, M.J., Colar, J.M., Geddes, T., Gold, J.M., Trivax, J.E. 2011. Changes in renal markers and acute kidney injury after marathon running. Nephrology 16, 194-199.

Nakano, T., Matsui, M., Inoue, I., Awata, T., Katayama, S., Murakoshi, T. 2011. Free immunoglobulin light chain: its biology and implications in diseases. Clin Chim Acta 412, 843849. 
Post-print, please cite as: Heaney, J.L.J., Phillips, A.C., Drayson, M.T. \& Campbell, J. (2016 in press). Serum free lights chains are reduced in endurance trained older adults: evidence that exercise training may reduce basal inflammation in older adults. Experimental Gerontology,

http://dx.doi.org/10.1016/i.exger.2016.02.011

Nelson, M.E., Rejeski, W.J., Blair, S.N., Duncan, P.W., Judge, J.O., King, A.C., Macera, C.A., Castaneda-Sceppa, C. 2007. Physical Activity and Public Health in Older Adults:

Recommendation From the American College of Sports Medicine and the American Heart Association. Circulation 116, 1094-1105.

Nieman, D.C., Nehlsen-Cannarella, S.L. 1991. The effects of acute and chronic exercise of immunoglobulins. Sports Med 11, 183-201.

Pratt, G. 2008. The evolving use of serum free light chain assays in haematology. Br J Haematol $141,413-422$.

Redegeld, F.A., Thio, M., Groot Kormelink, T. 2012. Polyclonal immunoglobulin free light chain and chronic inflammation. Mayo Clin Proc 87, 1032-1033.

Rehman, H.U., Masson, E.A. 2001. Neuroendocrinology of ageing. Age Ageing 30, 279-287.

Siegel, D., Bilotti, E., van Hoeven, K. 2009. Serum Free Light Chain Analysis for Diagnosis, Monitoring, and Prognosis of Monoclonal Gammopathies. Lab Med 40, 363-366.

Simpson, R.J., Lowder, T.W., Spielmann, G., Bigley, A.B., LaVoy, E.C., Kunz, H. 2012. Exercise and the aging immune system. Ageing Res Rev 11, 404-420.

Suki, W.N., Massry, S.G. (Eds.), 1998. Suki and Massry's Therapy of Renal Diseases and Related

Disorders. Kluer Academic Publishers

Tanaka, H., Monahan, K.D., Seals, D.R. 2001. Age-predicted maximal heart rate revisited. J Am Coll Cardiol 37, 153-156.

Touchberry, C.D., Ernsting, M., Haff, G., Kilgore, J.L. 2004. Training alterations in elite cyclists may cause transient changes in glomerular filtration rate. J Sports Sci Med 3, 28-36.

van der Heijden, M., Kraneveld, A., Redegeld, F. 2006. Free immunoglobulin light chains as target in the treatment of chronic inflammatory diseases. Eur J Pharmacol 533, 319-326.

Weinstein, J.R., Anderson, S. 2010. The Aging Kidney: Physiological Changes. Adv Chronic Kidney Dis 17, 302-307.

Weiskopf, D., Weinberger, B., Grubeck-Loebenstein, B. 2009. The aging of the immune system. Transplant International 22, 1041-1050. 
Post-print, please cite as: Heaney, J.L.J., Phillips, A.C., Drayson, M.T. \& Campbell, J. (2016 in press). Serum free lights chains are reduced in endurance trained older adults: evidence that exercise training may reduce basal inflammation in older adults. Experimental Gerontology,

http://dx.doi.org/10.1016/i.exger.2016.02.011

Woods, J.A., Wilund, K.R., Martin, S.A., Kistler, B.M. 2012. Exercise, inflammation and aging.

Aging Dis 3, 130-140.

\section{$\begin{array}{llll}\text { Sedentary } & \text { Physically active } & \text { Endurance Trained } & \text { All participants }\end{array}$}

\begin{tabular}{lcccc}
\hline Serum FLC concentration $(\mathrm{mg} / \mathrm{L})$ & & & \\
Kappa & $12.13 \pm 3.49$ & $12.39 \pm 2.89$ & $9.33 \pm 2.66$ & $11.26 \pm 3.24$ \\
Lambda & $12.19 \pm 3.51$ & $12.93 \pm 4.37$ & $7.52 \pm 1.89$ & $10.88 \pm 4.18$ \\
Sum & $24.32 \pm 6.50$ & $25.32 \pm 6.89$ & $16.85 \pm 4.35$ & $22.16 \pm 7.05$ \\
Difference & $-.07 \pm 2.58$ & $-.53 \pm 2.72$ & $1.81 \pm 1.55$ & $0.39 \pm 2.51$
\end{tabular}

Table 1. Mean \pm standard deviation baseline concentrations of serum free light chains, immunoglobulins, creatinine, estimated creatinine clearance rate $(\mathrm{eCrCl})$, estimated glomerular filtration rate (eGFR) and c-reactive protein (CRP) in sedentary, physically active and endurance trained older adults 
Post-print, please cite as: Heaney, J.L.J., Phillips, A.C., Drayson, M.T. \& Campbell, J. (2016 in press). Serum free lights chains are reduced in endurance trained older adults: evidence that exercise training may reduce basal inflammation in older adults. Experimental Gerontology,

Ratio http://dx.doi.org/10.1016/i.exger.2016.02.011

\begin{tabular}{|c|c|c|c|c|}
\hline \multicolumn{5}{|c|}{ Serum immunoglobulin concentration $(\mathrm{g} / \mathrm{L})$} \\
\hline $\lg G$ & $8.87 \pm 1.95$ & $9.52 \pm 2.25$ & $8.83 \pm 2.04$ & $9.10 \pm 2.07$ \\
\hline $\lg A$ & $1.99 \pm 0.96$ & $2.34 \pm 1.10$ & $1.91 \pm 0.66$ & $2.09 \pm 0.92$ \\
\hline $\lg M^{-}$ & $0.86 \pm 0.43$ & \multicolumn{3}{|c|}{ Sedentany $18 \pm 0.52$ Physically acti \pm 0.3 Endurance Traind \pm 0.47} \\
\hline Creatinine (AfGol/L) & $91.00 \pm 10.66$ & $67.90 \pm 5.1 .80 \pm 12.70$ & $69.31 \pm 4 . \operatorname{gg}_{3}^{*} .92 \pm 13.11$ & 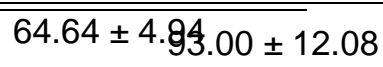 \\
\hline eCrCl (mL/rBMAl1.73m²) & $72.89 \pm 23.59$ & $26.62 \pm 36999 \pm 14.28$ & $25.46 \pm 2.64 .37 \pm 12.31$ & $22.75 \pm 2.68 .25 \pm 16.46$ \\
\hline \multicolumn{2}{|c|}{ 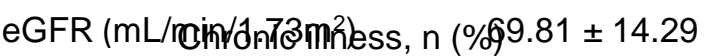 } & $4(36 \% 88.05 \pm 10.99$ & $3(19 \%) 69.70 \pm 9.05$ & $2(14 \%)^{69.13 \pm 11.25}$ \\
\hline CRP (mg/L)Chroni & $4(4 / 5)=$ & $6(55 \%) 1.45 \pm 1.06$ & $6(38 \%) \quad 1.55 \pm 1.61$ & $4(29 \%) 2.31 \pm 4.53$ \\
\hline
\end{tabular}

Table 2. Participant characteristics in sedentary, physically active and endurance trained older adults. Values are mean \pm standard deviation 
Post-print, please cite as: Heaney, J.L.J., Phillips, A.C., Drayson, M.T. \& Campbell, J. (2016 in press). Serum free lights chains are reduced in endurance trained older adults: evidence that exercise training may reduce basal inflammation in older adults. Experimental Gerontology, http://dx.doi.org/10.1016/i.exger.2016.02.011

Males, n (\%)

$6(54 \%)$

$11(69 \%)$

$10(71 \%)$

Moderate activity time per week (min)

$13.63 \pm 31.07^{\dagger \dagger}$

$277.88 \pm 167.29$

$51.53 \pm 61.68^{+\dagger}$

Vigorous activity time per week (min)
$0 \pm 0^{* * *}$

$22.50 \pm 50.60^{* * *}$

$233.27 \pm 129.64$

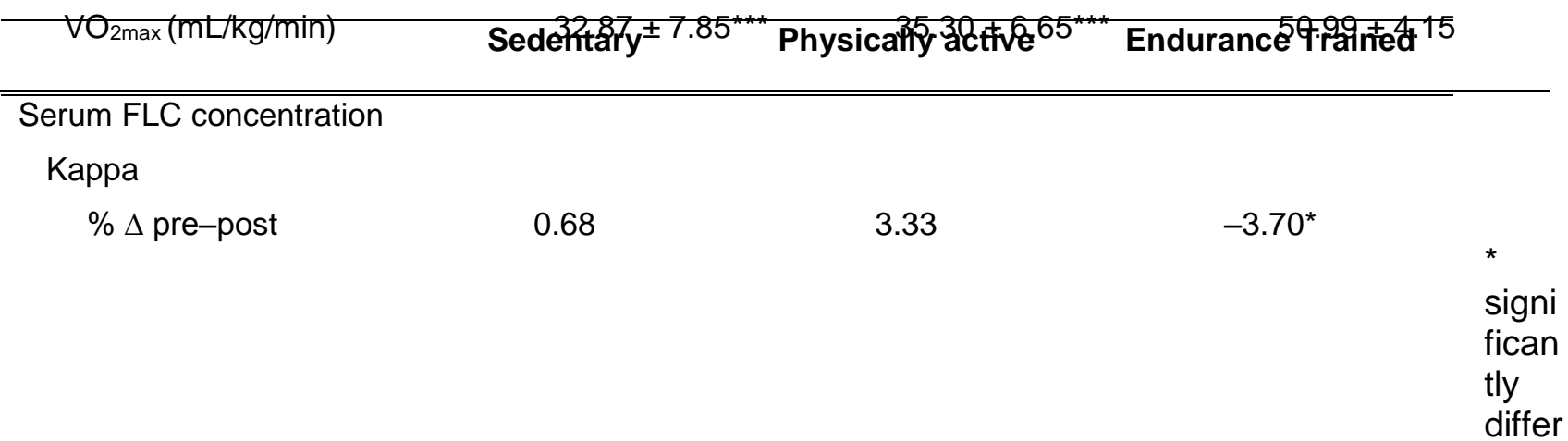

ent to endurance trained, ${ }^{*} p<.05,{ }^{* *} p<.01,{ }^{* *} p<.001$

†† significantly different to physically active, $p<.01$

Table 3. The effects of acute exercise on mean percentages changes in serum free light chains, immunoglobulins, creatinine and c-reactive protein (CRP) in sedentary, physically active and endurance trained older adults 
Post-print, please cite as: Heaney, J.L.J., Phillips, A.C., Drayson, M.T. \& Campbell, J. (2016 in press). Serum free lights chains are reduced in endurance trained older adults: evidence that exercise training may reduce basal inflammation in older adults. Experimental Gerontology, http://dx.doi.org/10.1016/i.exger.2016.02.011
$\% \Delta$ pre-1 h post
1.15
0.67
$-2.72$

Lambda

$\begin{array}{lccc}\% \Delta \text { pre-post } & -3.20 & 0.21 & 5.70^{\dagger} \\ \% \Delta \text { pre-1 h post } & -1.42 & -4.02 & 3.15\end{array}$

\begin{tabular}{|c|c|c|c|}
\hline \multicolumn{4}{|c|}{ Serum immunoglobulin concentration } \\
\hline \multicolumn{4}{|l|}{$\lg G$} \\
\hline$\% \Delta$ pre-post & 6.57 & 3.03 & 5.24 \\
\hline$\% \Delta$ pre-1 h post & 1.54 & -1.10 & -0.45 \\
\hline \multicolumn{4}{|l|}{$\lg A$} \\
\hline$\% \Delta$ pre-post & 6.63 & 4.05 & 5.90 \\
\hline$\% \Delta$ pre-1 h post & 0.69 & -0.29 & -0.32 \\
\hline \multicolumn{4}{|l|}{$\lg M$} \\
\hline$\% \Delta$ pre-post & 6.16 & 3.88 & 4.56 \\
\hline$\% \Delta$ pre-1 h post & -0.01 & -1.30 & -0.80 \\
\hline \multicolumn{4}{|l|}{ Creatinine } \\
\hline$\% \Delta$ pre-post & 2.49 & 2.21 & 2.11 \\
\hline$\% \Delta$ pre-1 h post & -3.78 & -2.07 & -5.31 \\
\hline \multicolumn{4}{|l|}{$\mathrm{eCrCl}$} \\
\hline$\% \Delta$ pre-post & -1.88 & -1.17 & -1.72 \\
\hline$\% \Delta$ pre-1 h post & 2.30 & 1.41 & 3.94 \\
\hline \multicolumn{4}{|l|}{ eGFR } \\
\hline$\% \Delta$ pre-post & -3.86 & -1.19 & -1.97 \\
\hline$\% \Delta$ pre-1 h post & 0.81 & 1.25 & 4.60 \\
\hline \multicolumn{4}{|l|}{ CRP } \\
\hline$\% \Delta$ pre-post & 9.53 & 4.89 & 4.58 \\
\hline$\% \Delta$ pre-1 h post & -0.30 & -2.36 & -4.12 \\
\hline
\end{tabular}

* significantly different to physically active, $p<.05$

† significantly different to sedentary, $p<.05$ 
Post-print, please cite as: Heaney, J.L.J., Phillips, A.C., Drayson, M.T. \& Campbell, J. (2016 in press). Serum free lights chains are reduced in endurance trained older adults: evidence that exercise training may reduce basal inflammation in older adults. Experimental Gerontology, http://dx.doi.org/10.1016/i.exger.2016.02.011
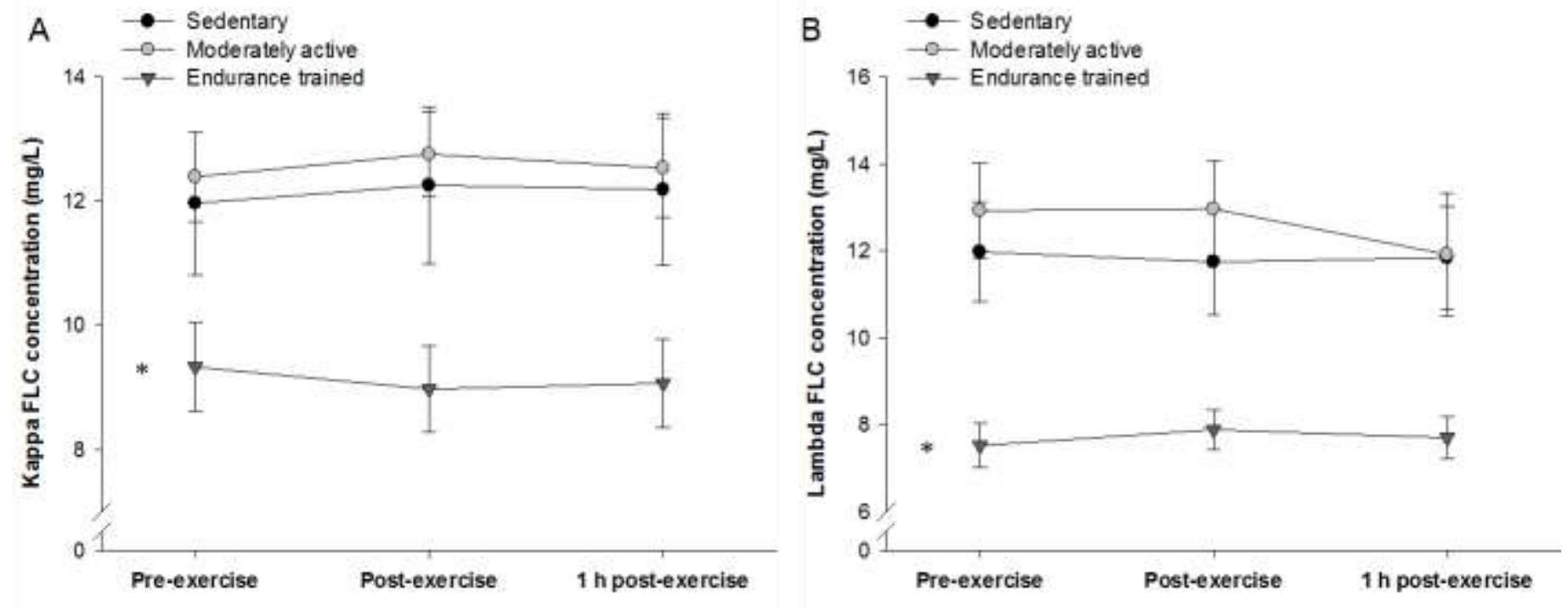

Figure 1. Kappa $(A)$ and lambda $(B)$ free light chain $(F L C)$ concentrations in serum in response to submaximal exercise in sedentary, moderately active and endurance trained older adults * Indicates a significant main effect of group: endurance trained older adults had lower overall kappa and lambda FLC concentrations in serum compared with sedentary and moderately trained groups, $p<.05$. There were no significant effects of time or group $\mathrm{x}$ time interactions. Values are mean \pm SEM 
Post-print, please cite as: Heaney, J.L.J., Phillips, A.C., Drayson, M.T. \& Campbell, J. (2016 in press). Serum free lights chains are reduced in endurance trained older adults: evidence that exercise training may reduce basal inflammation in older adults. Experimental Gerontology,
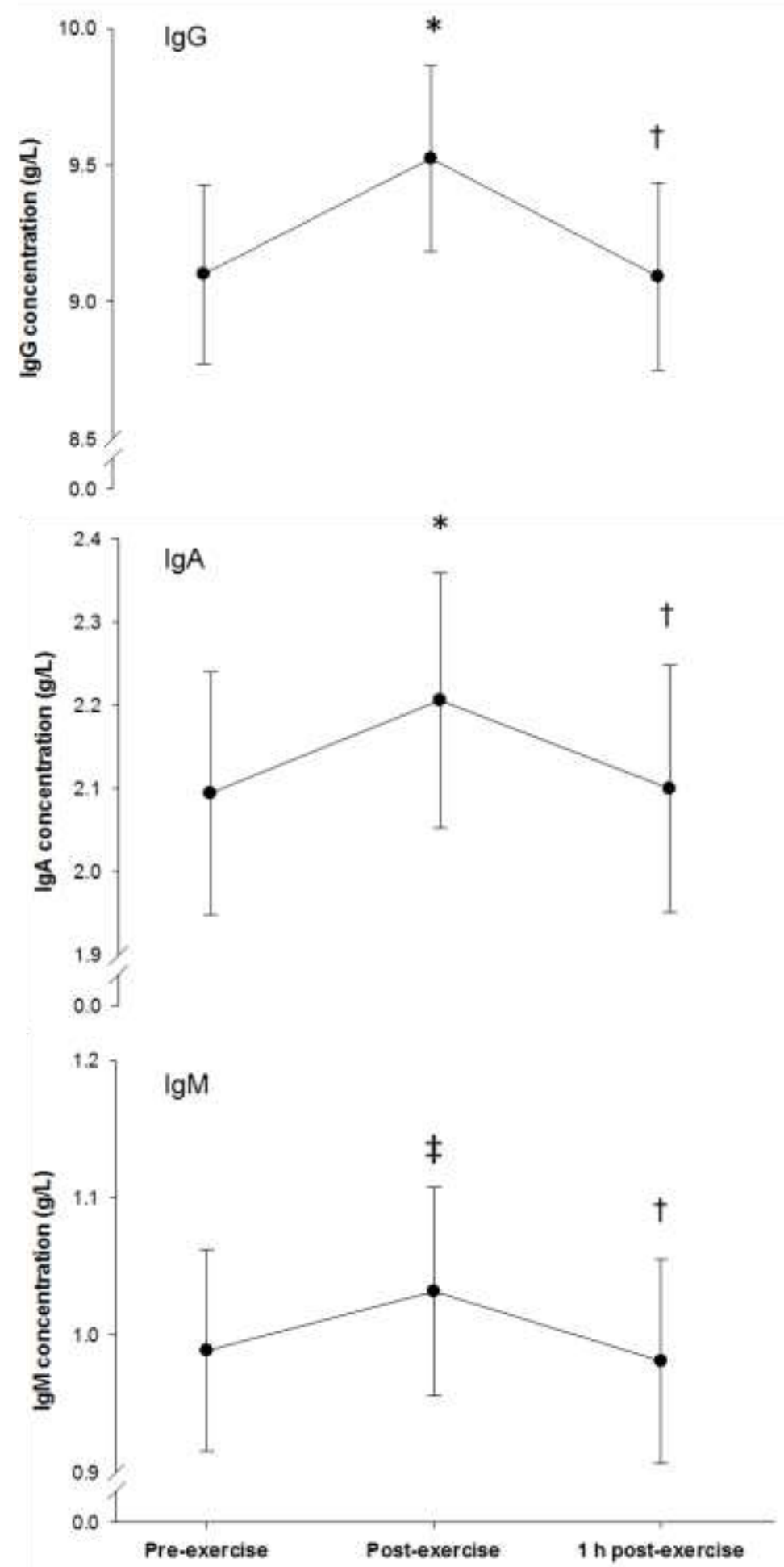

Figure 2. $\lg G, \lg A$ and $\lg M$ concentrations in serum in response to submaximal exercise in older adults

* Indicates a significant difference to pre exercise: $\lg G$ and $\lg A$ significantly increased pre-post exercise $(p<.01)$.

$\ddagger \lg M$ increased pre-post exercise but was no longer significant after controlling for plasma volume change $(\mathrm{P} \vee \Delta)$

† All immunoglobulins decreased between postand $1 \mathrm{~h}$ post exercise; these findings did not remain significant after controlling for $P V \Delta$, reflecting rehydration during this period Values are mean \pm SEM 
Post-print, please cite as: Heaney, J.L.J., Phillips, A.C., Drayson, M.T. \& Campbell, J. (2016 in press). Serum free lights chains are reduced in endurance trained older adults: evidence that exercise training may reduce basal inflammation in older adults. Experimental Gerontology, http://dx.doi.org/10.1016/i.exger.2016.02.011

A

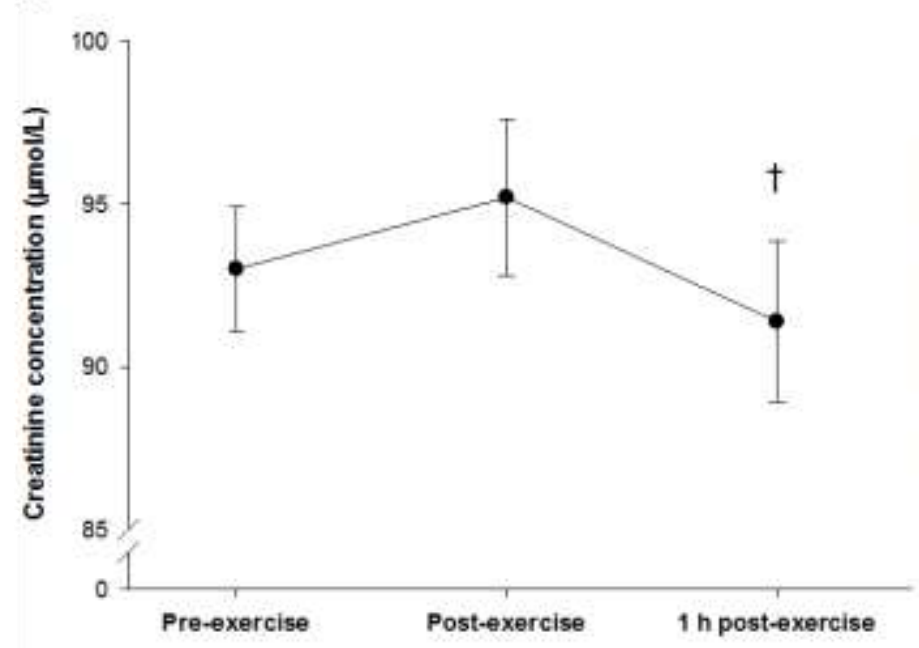

\section{B}

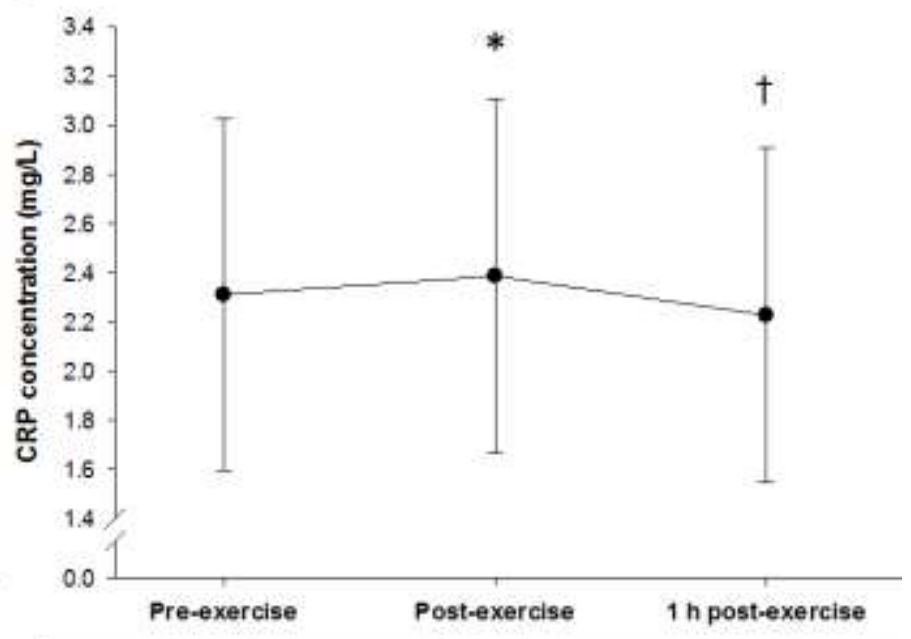

Figure 3. Creatinine (A) and c-reactive protein (CRP, B) concentrations in response to submaximal exercise in older adults. $*$ Indicates a significant increase in CRP pre- to post-exercise , $p<0.05$; † CRP and creatinine decreased between post- and $1 \mathrm{~h}$ post-exercise but did not remain significant after controlling for changes in plasma volume, reflecting rehydration during this period. Values are mean \pm SEM 